\title{
Velocity difference statistics in turbulence
}

\author{
Sunghwan Jung* and Harry L. Swinney \\ Center for Nonlinear Dynamics and Department of Physics, \\ The University of Texas at Austin, Austin, Texas 78712 USA.
}

(Dated: August 23, 2018)

\begin{abstract}
We unify two approaches that have been taken to explain the non-Gaussian probability distribution functions (PDFs) obtained in measurements of longitudinal velocity differences in turbulence, and we apply our approach to Couette-Taylor turbulence data. The first approach we consider was developed by Castaing and coworkers, who obtained the non-Gaussian velocity difference PDF from a superposition of Gaussian distributions for subsystems that have a particular energy dissipation rate at a fixed length scale [Castaing et al., Physica D 46, 177 (1990)]. Another approach was proposed by Beck and Cohen, who showed that the observed PDFs can be obtained from a superposition of Gaussian velocity difference PDFs in subsystems conditioned on the value of an intensive variable (inverse "effective temperature") in each subsystem [Beck and Cohen, Physica A 322, 267 (2003)]. The intensive variable was defined for subsystems assuming local thermodynamic equilibrium, but no method was proposed for determining the size of a subsystem. We show that the Castaing and Beck-Cohen methods are related, and we present a way to determine subsystem size in the Beck-Cohen method. The application of our approach to Couette-Taylor turbulence (Reynolds number 540 000) yields a log-normal distribution of the intensive parameter, and the resultant velocity difference PDF agrees well the observed non-Gaussian velocity difference PDFs.
\end{abstract}

PACS numbers: 47.27.Ak, 47.27.-i, 05.20.-y, 47.27.Jv

\section{INTRODUCTION}

In Kolmogorov's 1941 theory (K41), the energy in fully developed three-dimensional turbulence cascades from large scales to small scales where it is dissipated [1]. Turbulence in the cascade (the inertial range) is characterized by the probability distribution function (PDF) $P\left(\delta v_{r}\right)$ for longitudinal velocity differences over a distance $\left.r, \delta v_{r}(x)=\hat{e}_{r} \cdot[\vec{v}(x+r)-\vec{v}(x)]\right)$, where $\hat{e}_{r}$ is the direction of separation [2]. For $r$ approaching the integral scale where energy is injected, the PDF is Gaussian, while in the inertial range extending down to the dissipation scale $\eta$, intermittent large fluctuations lead to a non-Gaussian PDF with approximately exponential tails 3].

Kolmogorov assumed a constant energy dissipation rate per unit volume, $\varepsilon$ [1]. In 1944 Landau [4] suggested that fluctuations of $\varepsilon$ averaged at scale $r$, $\varepsilon_{r}(\vec{x}, t)\left(=\int_{\vec{x}}^{\vec{x}+\vec{r}} \varepsilon\left(\vec{x}^{\prime}, t\right) d \vec{x}^{\prime}\right)$ play a key role in turbulence. Such fluctuations were subsequently observed in many experiments [5, 6, 7]. In 1962 Kolmogorov [8] and Obukhov [9] proposed a log-normal distribution of $\varepsilon_{r}$ in the inertial range. The log-normal distribution was obtained in subsequent experiments and numerical simulations $\varepsilon_{r}$ 10, 11, 12, 13]. The non-Gaussian PDF of $\delta v_{r}$ and the $\log$-normal PDF of $\varepsilon_{r}$ characterize turbulent flows.

Different approaches have been taken by Castaing et al. 14 and by Beck and Cohen 15] to understand the non-Gaussian $P\left(\delta v_{r}\right)$. Castaing et al. assumed that sub-

*Electronic address: sunnyjsh@chaos.utexas.edu systems have different values of $\varepsilon_{r}$, but the subsystems have Gaussian PDFs of $\delta v_{r}$; this assumption is supported by experiments.

Beck and Cohen took a statistical mechanics approach, assuming that subsystems have a well-defined "effective temperature", which for turbulent flow is identified with the variance of $\delta v_{r}$. The resultant $P\left(\delta v_{r}\right)$ depends on the statistics of the distribution for the inverse effective temperatures in the subsystems. This dependence of the statistical distribution $P\left(\delta v_{r}\right)$ on the statistical distribution of subsystems led Beck and Cohen to call their approach superstatistics [15].

In this paper we note that the approaches of Castaing et al. and Beck and Cohen are both based on Bayes' theorem,

$$
P(x)=\int P(x \mid y) P(y) d y,
$$

which is used to obtain the non-Gaussian $P\left(\delta v_{r}\right)$ from a conditional mixing of Gaussian PDFs in subsystems. However, the subsystems are chosen differently in the two approaches.

We propose a method that does not require a determination of $\varepsilon_{r}$ from experimental data, nor does it require a fitting parameter to obtain the effective temperature PDF. We show that subsystems with Gaussian statistics can be chosen by examining moments of velocity difference distributions in the subsystems. Our method, which involves no fitting parameters, leads to predictions for the non-Gaussian $P\left(\delta v_{r}\right)$ that are in accord with data for turbulent Couette-Taylor flow [16].

In Section III we present the Castaing et al. and Beck and Cohen methods, and in Section III we describe the Couette-Taylor experiments and present results for 
$P\left(\delta v_{r}\right)$. Section $\llbracket$ shows how subsystems can be systematically chosen to obtain a prediction for $P\left(\delta v_{r}\right)$. The conclusions are presented in Section V

\section{THEORY}

\section{A. Method of Castaing et al.}

Castaing et al. 14] started with the observation from their experiments that that velocity difference distributions for a given $\varepsilon_{r}$ are Gaussian, and that $\varepsilon_{r}$ is described by a log-normal distribution [14, 17, 18, 19, 20, 21]. The log-normal distribution for $\varepsilon_{r}$ has also been obtained for $\varepsilon_{r}$ in other experiments on fully developed turbulence [18, 22, 23, 24], and in analyses of images of cloud patterns 25], effective temperature fields in turbulence 26], and magnetic fields in solar winds [27].

To describe the evolution of $P\left(\delta v_{r}\right)$ from Gaussian at large scales to non-Gaussian at small scales [28, 29, 30], Castaing et al. proposed [14, 17]

$$
P\left(\delta v_{r}\right)=\int P\left(\varepsilon_{r}\right) P\left(\delta v_{r} \mid \varepsilon_{r}\right) d \varepsilon_{r} .
$$

The conditional PDF $P\left(\delta v_{r} \mid \varepsilon_{r}\right)$ in Eq. (2) is assumed to be a Gaussian distribution, $P\left(\delta v_{r} \mid \varepsilon_{r}\right)=e^{-\left(\delta v_{r}\right)^{2} /\left(r \varepsilon_{r}\right)^{2 / 3}}$, in accord with experimental observations 31, 32, 33. Kolmogorov [8], Obukhov [9] and Castaing [14] assumed a $\log$-normal distribution of $\varepsilon_{r}$,

$$
P\left(\varepsilon_{r}\right)=\frac{1}{\lambda_{\varepsilon}(2 \pi)^{1 / 2} \varepsilon_{r}} \exp \left(-\frac{\left(\ln \varepsilon_{r}-m_{\varepsilon}\right)^{2}}{2 \lambda_{\varepsilon}^{2}}\right),
$$

where $m_{\varepsilon}$ and $\lambda_{\varepsilon}$ are respectively the mean and the standard deviation of $\ln \varepsilon_{r}$.

A difficulty in applying the approach of Castaing et al. is that energy dissipation rate at length scale $r, \varepsilon_{r}$, is not directly measured in experiments. By assuming homogeneous and isotropic conditions, $\varepsilon_{r}(x)$ is defined as $15 \nu \int_{x}^{x+r}(\partial v / \partial x)^{2} d x$. In practice, $\varepsilon_{r}$ is determined from time series data,

$$
\varepsilon_{r}=\frac{15 \nu}{(\Delta x)^{2}} \sum_{i=1}^{N-1}\left[v\left(x_{i+1}\right)-v\left(x_{i}\right)\right]^{2},
$$

where $\Delta x\left(\equiv x_{2}-x_{1}\right)$ is the sampling separation the summation $i$ is over subsystems and $x_{N}-x_{1}=r$ [33, 34, 35]. Even with this assumption, determination of $\varepsilon_{r}(x)$ is difficult because of errors in evaluating the derivative from velocity data. Further error arises from the application of the Taylor frozen hypothesis at high frequencies 24, 36, 37, 38, 39, 40, 41, 42, 43, 44].

\section{B. Superstatistics of Beck and Cohen}

Beck and Cohen's statistical approach considers a system far from thermodynamic equilibrium to consist of subsystems in local thermodynamic equilibrium [15]. Each subsystem has a well-defined "effective temperature", but the subsystem effective temperatures need not be the same since the whole system is not in equilibrium. Beck and Cohen identify $\left(\delta v_{r}\right)^{2}$ with the kinetic energy of eddies of size $r, E\left(\delta v_{r}\right)=\frac{1}{2}\left(\delta v_{r}\right)^{2}$, and the variance of $\delta v_{r}$ is identified with an inverse effective temperature $\beta$ [45], given for a subsystem of size $d$ by

$$
\beta_{d}=\frac{1}{\left\langle\left(\delta v_{r}\right)^{2}\right\rangle_{d}-\left(\left\langle\delta v_{r}\right\rangle_{d}\right)^{2}}
$$

where $\langle\cdot\rangle_{d}$ is an average over the size $d$. Then we have

$$
P\left(\delta v_{r}\right)=\int_{0}^{\infty} P\left(\beta_{d}\right) P\left(\delta v_{r} \mid \beta_{d}\right) d \beta_{d} .
$$

where $P\left(\beta_{d}\right)$ is the distribution of inverse effective temperature in subsystems of size $d$.

A particular choice of $P\left(\beta_{d}\right)$, the $\chi^{2}$ distribution, leads to the distribution associated with the nonextensive statistical mechanics of Tsallis, $P(E)=(1+\beta(q-$ $1) E)^{-1 /(q-1)}$, where $q$ is a parameter characterizing the nonextensivity $[S(1+2)=S(1)+S(2)+(1-q) S(1) \cdot S(2)$, where $S$ is entropy function.] [15, 46]. A phenomenology similar to Beck and Cohen's was used in earlier oceanographic analysis that described the global non-Gaussian distribution of ocean surface velocity as a mixture of local Gaussians with $\chi^{2}$-distributed variance [47, 48]. The method of Beck and Cohen has been applied to fully developed turbulence [49, 50] by introducing a fitting parameter to determine the PDF of inverse effective temperature, rather than by directly measuring the PDF of inverse effective temperature.

The Beck-Cohen method requires that the size $d$ should be large compared to the distance $r$ separating two points, and $d$ should also be large enough so the subsystems contain enough data points to yield good statistics, but $d$ must also be small enough so that subsystems are each described by a Gaussian distribution. Beck determined the size of $d$ using a fitting parameter involving the kurtosis of $P\left(\delta v_{r}\right)$ [50].

\section{Unified view of PDFs}

The Castaing and Beck-Cohen methods are similar except in the way they divide a system into subsystems. Castaing et al. sample velocity differences conditioned by the averaged energy dissipation rate $\varepsilon_{r}$, while Beck and Cohen use velocity differences conditioned by the inverse effective temperature $\beta_{d}$. Castaing et al. need one fixed length scale, the separation distance $r$ between two points; $\delta v_{r}$ and $\varepsilon_{r}$ are defined at this scale and are related through Bayes' theorem. The Beck-Cohen method involves two length scales, the distance $r$ separating two points and the size $d$ of the subsystems in the statistical analysis. 
The Castaing and Beck-Cohen methods can be connected if the two conditioning variables $\left(\varepsilon_{r}\right.$ and $\left.\beta_{d}\right)$ are correlated. Using Eq. (2) and Bayes' theorem, we convert Castaing's method into Beck-Cohen's method,

$$
\begin{aligned}
& P\left(\delta v_{r}\right)=\int_{0}^{\infty} P\left(\delta v_{r} \mid \varepsilon_{r}\right) P\left(\varepsilon_{r}\right) d \varepsilon_{r} \\
= & \int_{0}^{\infty} \int_{0}^{\infty} P\left(\delta v_{r} \mid \beta_{d}\right) P\left(\beta_{d} \mid \varepsilon_{r}\right) d \beta_{d} P\left(\varepsilon_{r}\right) d \varepsilon_{r} \\
= & \int_{0}^{\infty} P\left(\delta v_{r} \mid \beta_{d}\right)\left[\int_{0}^{\infty} P\left(\beta_{d} \mid \varepsilon_{r}\right) P\left(\varepsilon_{r}\right) d \varepsilon_{r}\right] d \beta_{d} \\
= & \int_{0}^{\infty} P\left(\delta v_{r} \mid \beta_{d}\right) P\left(\beta_{d}\right) d \beta_{d} .
\end{aligned}
$$

Now, let's assume a log-normal distribution of $\beta_{d}$ at the fixed $\varepsilon_{r}$,

$$
P\left(\beta_{d} \mid \varepsilon_{r}\right) \propto \frac{1}{\beta_{d}} \exp \left[-\frac{\left(\ln \beta_{d}-a \ln \varepsilon_{r}\right)^{2}}{2 \lambda_{t}^{2}}\right],
$$

where $\lambda_{t}$ is the standard deviation of $\ln \beta_{d}$ conditioned to $\varepsilon_{r}$, and $a$ is a parameter. Using Eqs. (3), (7), and (9), we have

$$
\begin{aligned}
P\left(\delta v_{r}\right) & \propto \int_{0}^{\infty} P\left(\delta v_{r} \mid \beta_{d}\right) \int_{0}^{\infty} \exp \left(-\frac{\left(\ln \beta_{d}-a \ln \varepsilon_{r}\right)^{2}}{2 \lambda_{t}{ }^{2}}\right) \\
& \times \exp \left(-\frac{\left(\ln \varepsilon_{r}-m_{\varepsilon}\right)^{2}}{2 \lambda_{\varepsilon}^{2}}\right) d\left(\ln \varepsilon_{r}\right) d\left(\ln \beta_{d}\right) \\
\propto & \int_{0}^{\infty} P\left(\delta v_{r} \mid \beta_{d}\right) \exp \left(-\frac{\left(\ln \beta_{d}-m\right)^{2}}{\lambda_{\varepsilon}^{2} \lambda_{t}^{2}}\right) d\left(\ln \beta_{d}\right) .
\end{aligned}
$$

Thus with the assumption of a log-normal distribution of $\beta_{d}$ conditioned on $\varepsilon_{r}$, we have that Castaing's method is equivalent to Beck-Cohen's method. In SectionIVD the log-normal PDF of $P\left(\beta_{d} \mid \varepsilon_{r}\right)$ is verified in experiments.

\section{EXPERIMENT}

We describe here an experiment on turbulent CouetteTaylor flow by Lewis and Swinney [16, 51], and in the next section we will analyze data from this experiment to deduce $P(\beta)$ and a prediction for $P\left(\delta v_{r}\right)$. The fluid was contained in the annular region between two concentric cylinders with an inner radius of $b=22.085 \mathrm{~cm}$ and an outer radius of $a=15.999 \mathrm{~cm}$; thus the ratio of inner to outer radius was 0.724 . The height of the annulus was $69.5 \mathrm{~cm}$, which yields a value of 11.4 for the ratio of height to the gap. The inner cylinder angular rotation rate $\Omega$ was $8 \times 2 \pi \mathrm{rad} / \mathrm{s}$; the outer cylinder was at rest. The ends of the annulus rotated at the same rate as the inner cylinder. The fluid was water with a viscosity $\nu$ of $0.00968 \mathrm{~cm}^{2} / \mathrm{s}$ at the working effective temperature. Defining the Reynolds number as $R e=\Omega a(b-a) / \nu$ yields for the Reynolds number 540000 [16].

A hot film probe was used to measure the time dependence of the azimuthal component of the velocity in the

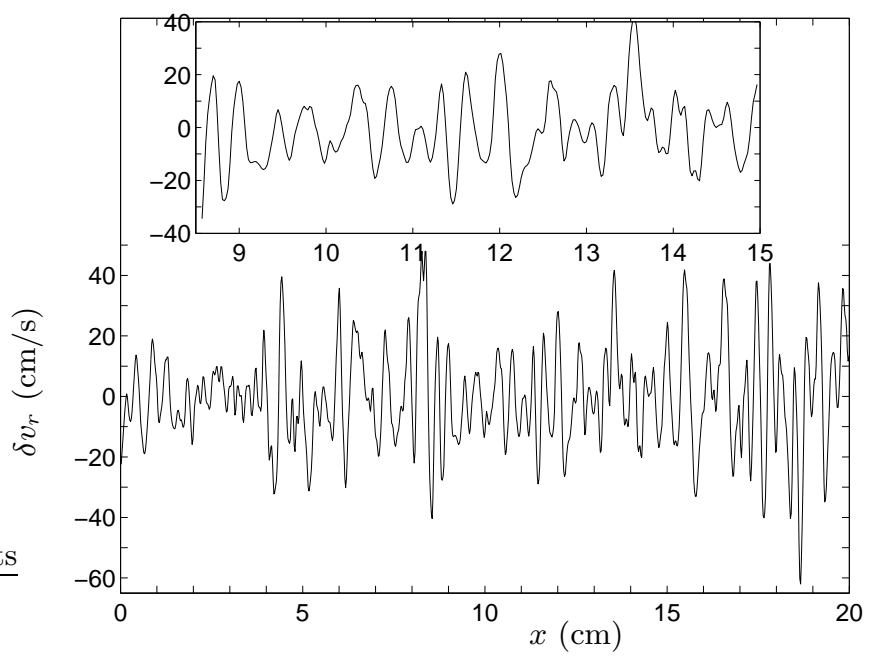

FIG. 1: An example of the Couette-Taylor velocity difference data, obtained by subtracting velocities at two points with a separation $r=46 \eta=0.134 \mathrm{~cm}$, where $\eta$ is the Kolmogorov length scale. The inset shows the velocity differences on a finer length scale.

center of the gap at a distance $4.35 \mathrm{~cm}$ above mid-height of the annulus. The Taylor frozen turbulence hypothesis was used to convert the velocity time series data to velocity field data. The turbulent intensity (the ratio of the root mean squared velocity to the mean velocity) was less than $6 \%$.

The uncertainties shown on our graphs correspond to the standard deviation of 20 independent experiments. The velocity measurements were made with a sampling rate 2500 times the inner cylinder rotation frequency; this corresponds to a spatial separation of $0.017 \mathrm{~cm}$ between successive velocity values. The longitudinal velocity differences $\delta v_{r}$ that we analyze are for points separated by a small distance, $r=0.134 \mathrm{~cm}$, where the probability distribution function has approximately exponential tails [16]. An example of the measurements of $\delta v_{r}(t)$ is shown in Fig. 1] The separation $r=0.134 \mathrm{~cm}$ corresponds to $46 \eta$, where $\eta$ is Kolmogorov scale 16]. (The Kolmogorov dissipation scale was obtained by calculating the dissipation from energy spectra: $\eta \equiv(\nu / \varepsilon)^{1 / 4}$, where the dissipation rate is given by $\varepsilon=15 \nu \int k^{2} E(k) d k$ [16].) The window size $d$ we use for determining the local inverse effective temperature $\beta$ is typically $0.9 \mathrm{~cm}$, nearly an order of magnitude larger than the value of $r$.

\section{RESULTS}

\section{A. Probability density function of inverse effective temperature}

Several distributions for inverse effective temperature $\beta_{d}$ have been discussed by Beck and Cohen [15]. Here we 

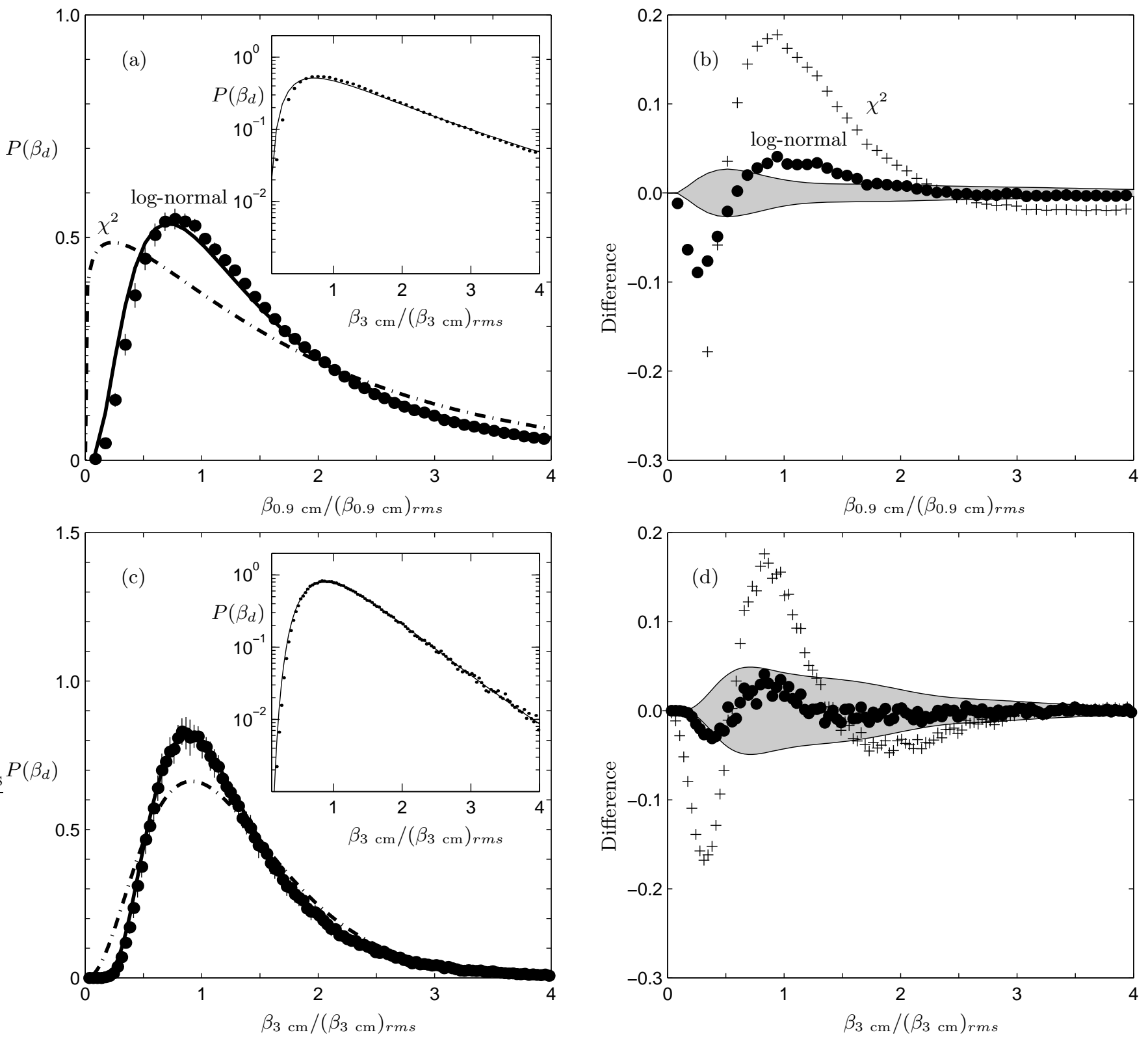

FIG. 2: Comparison of $\chi^{2}$ and log-normal distributions to the experimental distribution for inverse effective temperature in subsystems of size (a) $d=0.9 \mathrm{~cm}$ and (c) $d=3 \mathrm{~cm}$. The dash-dotted lines represent the $\chi^{2}$ distribution, and the solid line represents the log-normal distribution; both have the same mean and variance as the 20 independent experiments (error bars correspond to one standard deviation). The panels on the right, (b) and (d), show the difference between the experimental PDF for $\beta_{d}$ and the $\chi^{2}$ (plus signs) and log-normal (bullets) distributions for (a) $d=0.9 \mathrm{~cm}$ and (c) $d=3 \mathrm{~cm}$. The shaded area represents the experimental uncertainty (standard deviation of 20 experiments).

consider the $\log$ normal and $\chi^{2}$ distributions, which are most applicable to turbulent flow. Due to multiplicative processes in turbulence, the log-normal distribution is often observed for positive-definite quantities ( $\operatorname{such}$ as $\varepsilon_{r}$ ) 14, 17, 18, 19, 20, 21]. A log-normally distributed $\beta_{d}$ is given by

$$
P\left(\beta_{d}\right)=\frac{1}{s(2 \pi)^{1 / 2} \beta_{d}} \exp \left(-\frac{\left(\log \beta_{d}-m\right)^{2}}{2 s^{2}}\right)
$$

where $s=\sqrt{\ln \left(1+\sigma_{\beta_{d}}^{2} / \bar{\beta}_{d}^{2}\right)}$ and $m=$ $\log \left({\overline{\beta_{d}}}^{2} / \sqrt{{\overline{\beta_{d}}}^{2}+\sigma_{\beta_{d}}^{2}}\right)$ are parameters, and $\overline{\beta_{d}}$ and $\sigma_{\beta_{d}}$ are respectively the mean and standard deviation of $\beta_{d}$.

The $\chi^{2}$ distribution of $\beta_{d}$ is given by

$$
P\left(\beta_{d}\right)=\frac{1}{\beta_{d} \Gamma(c)}\left(\frac{\beta_{d}}{b}\right)^{c} \exp \left(-\frac{\beta_{d}}{b}\right)
$$




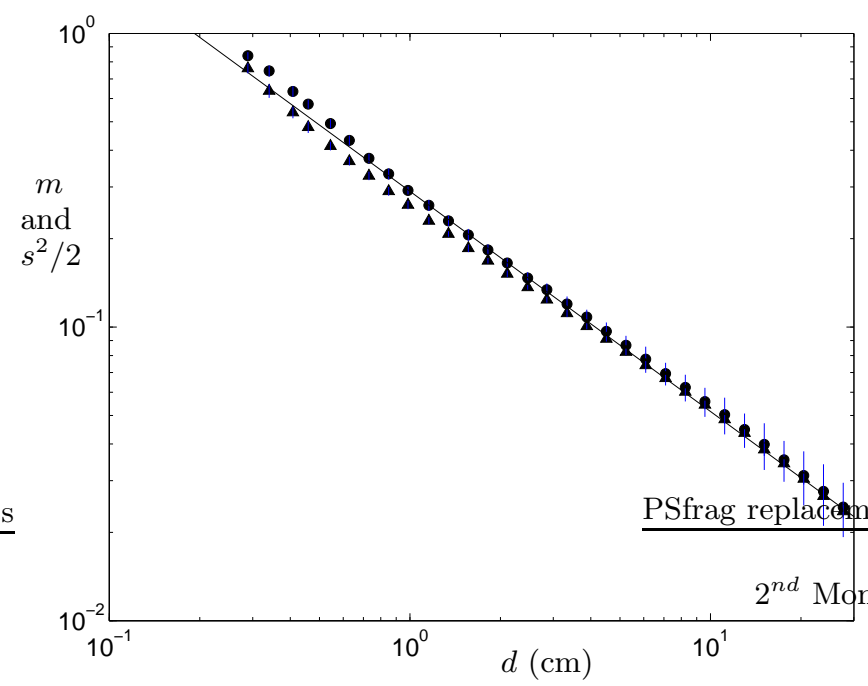

FIG. 3: The parameters $\frac{s^{2}}{2}$ (circles) and $m$ (triangles), obtained from fits of the inverse effective temperature $\beta_{d}$ (deduced from Couette-Taylor turbulence data) to a log-normal distribution, as a function of subsystem size $d$ (see Eq. (11). ( $s$ and $m$ are the variance and mean of logarithmic inverse effective temperature.) The parameters $\frac{s^{2}}{2}$ and $m$ are approximately equal (see text) and are described by a power law, $m \propto d^{-3 / 4}$ (solid line).

where $c={\overline{\beta_{d}}}^{2} / \sigma_{\beta_{d}}^{2}$ and $b=\sigma_{\beta_{d}}^{2} / \overline{\beta_{d}}$ and $\Gamma$ is the gamma function. The $\chi^{2}$ distribution has been observed in recent measurement of wind turbulence [52]. The statistical properties of different distributions are discussed in [15.

The experimental PDF for $\beta_{d}$ is compared in Fig. 2 with a log-normal distribution and with a $\chi^{2}$ distribution for two subsystem sizes $d, 0.9 \mathrm{~cm}$ and $3 \mathrm{~cm}$. The mean $\overline{\beta_{d}}$ and variance $\sigma_{\beta_{d}}^{2}$ of the inverse effective temperature determine the parameters $s, m, b$ and $c$. For small $d$, the log-normal and $\chi^{2}$ differ significantly, but for large $d$ they become closer together [Fig. 2(c) and (d)]. The decrease in variance of $\beta_{d}$ with increasing $d$ is similar to decrease observed in the variance of $\varepsilon_{r}$ with increasing $r$ [53].

The difference between the PDF of $\beta_{d}$ from experiment and the $\chi^{2}$ and log-normal distributions is shown in Fig. 2(b) and (d). For $d=0.9 \mathrm{~cm}$, the log-normal distribution fits the data within the experimental uncertainty except small $\beta_{d}$ regions, while the $\chi^{2}$ distribution deviates from the observations by an amount that is large compared to the uncertainty. For $d=3 \mathrm{~cm}$, the log-normal distribution fits the distribution of $\beta_{d}$ whereas the $\chi^{2}$ distribution does not.

The log normal distribution (11) involves two parameters, $s$ and $m$, which depend on subsystem size, as shown in Fig. 3 This figure suggests a relationship between $s$ and $m, m=\frac{s^{2}}{2}$, which is supported by a calculation in Castaing et al. (see Section 4.3.1 in [14]).

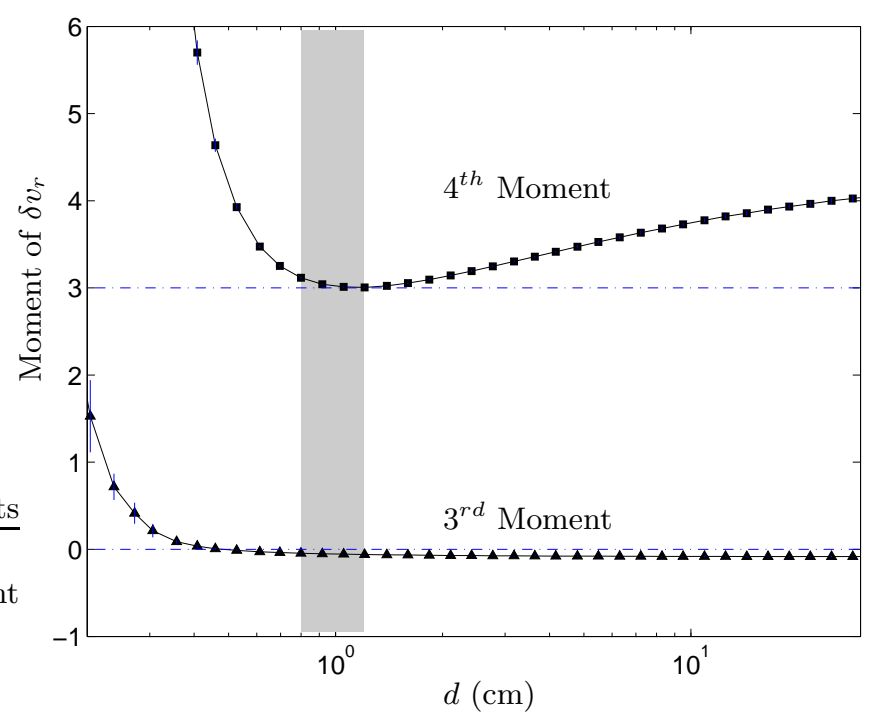

FIG. 4: The dependence of the third and fourth moments of $P\left(\delta v_{r}\right)$ on the size $d$ of the subsystems. For sufficiently small $d, P\left(\delta v_{r}\right)$ should be Gaussian, which means the values of the third and fourth moments should have the values zero and three, respectively. We find that at $d \approx 0.9 \mathrm{~cm}$, the conditional distribution Eq. (11) is close to Gaussian (see text).

\section{B. Conditional probability and the proper subsystem size}

In the statistical approach of Beck and Cohen, the subsystem size $d$ should be sufficiently small so that $P\left(\beta_{d}\right)$ is Gaussian, corresponding to local thermodynamic equilibrium in the subsystems. However, in practice the $d \longrightarrow 0$ limit is inaccessible because as $d$ becomes very small, the number of data points becomes too small to allow accurate determination of the variance of $\beta_{d}$. So what is optimal choice of $d$ ? We address this question by examining the third moment (skewness) and fourth moment (kurtosis) of $\delta v_{r}$, which should be equal respectively to zero and three for a Gaussian distribution. In principle we could also examine fifth and higher moments, but because of the sensitivity of the higher moments to noise, we limit our considerations to the third and fourth moments. Plotting the third and fourth moments as a function of $d$, as shown in Fig. 4 we find that the optimal value of $d$ for our data is $1.0-1.2 \mathrm{~cm}$, which is the only range in which the kurtosis is approximately given by the value for a Gaussian. The skewness is small and negative for $d>0.5 \mathrm{~cm}$, but becomes strongly positive for $d<0.5 \mathrm{~cm}$, reflecting a cascade of energy to smaller length scales. We conclude that $d=0.9 \mathrm{~cm}$ is the optimal subsystem size for our data. 

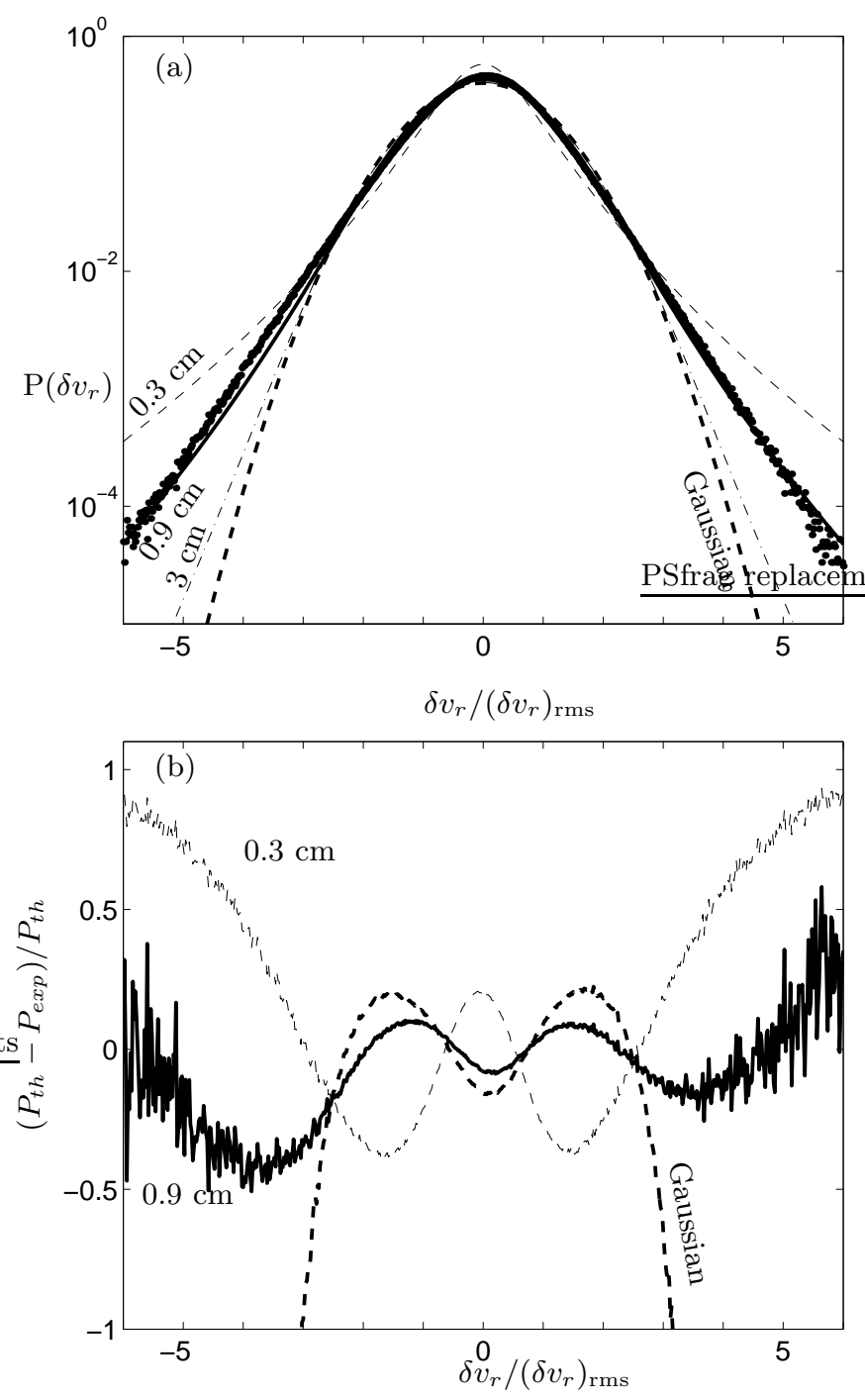

FIG. 5: Comparison experimental results (dots) for $P\left(\delta v_{r}\right)$ with the prediction of the Beck-Cohen method for a subsystem with the optimal size of $0.9 \mathrm{~cm}$ (bold line) on semi-log scale in (a) and relative error between theoretical and experimental values in (b). For comparison, we also show in (a) and (b) the predictions for subsystems of size $0.3 \mathrm{~cm}$ (thin dashed line) and $3 \mathrm{~cm}$ (thin dash-dot line) and a Gaussian distribution (dashed line).

\section{Probability distribution of $\delta v_{r}$}

We found a log-normal distribution of $\beta_{d}$ fits the turbulence data over a wide range in $d$ (Section IVA). With the $\log$ normal distribution of $\beta_{d}$ for the optimal value of $d(0.9 \mathrm{~cm}$, Fig. 4 4) and the conditional Gaussian distribution of $\delta v_{r}$ for that $\beta_{d}$, we obtain the probability distribution of $\delta v_{r}$ by the method of Beck and Cohen,

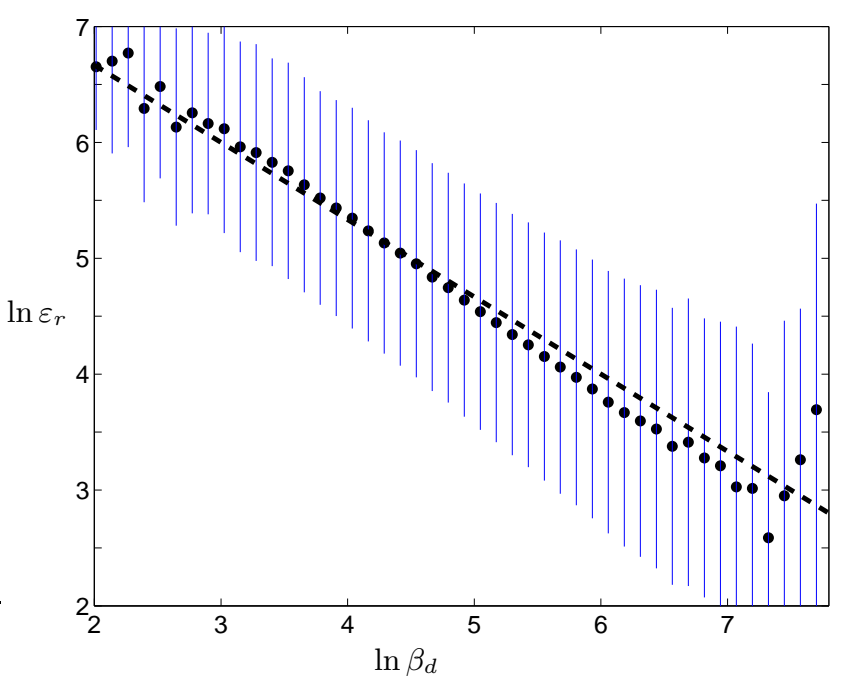

FIG. 6: The relation between $\beta_{d}$ and $\varepsilon_{r}$. The solid vertical lines represent standard deviations at a fixed $\beta_{d}$ and the dots represent the mean values. The dashed line is $\beta_{d} \propto\left(\varepsilon_{r}\right)^{-2 / 3}$.

$$
\begin{aligned}
P\left(\delta v_{r}\right)= & \frac{1}{2 \pi s} \int_{0}^{\infty} d \beta_{d} \beta_{d}^{-1 / 2} \exp \left(-\frac{\left(\log \beta_{d}-m\right)^{2}}{2 s^{2}}\right) \\
& \times \exp \left(-\frac{1}{2} \beta_{d}\left(\delta v_{r}\right)^{2}\right)
\end{aligned}
$$

where $s$ and $m$ are determined from experiment for the optimal subsystem size $d$. There is no explicit form for the improper integral in Eq. (13) so we evaluate the integral numerically, using the limits $\left(\left[\min \beta_{d}, \max \beta_{d}\right]\right)$ measured in experiments instead of the theoretical integral domain, $[0, \infty)$.

The results for $P\left(\delta v_{r}\right)$ obtained by numerical integration of (13) are shown in Fig. [5] The data are described much better by the predicted probability distribution than by a Gaussian. The observed approximate power law tails are similar to the predicted distribution function.

\section{Castaing and Beck-Cohen methods}

If the two conditioning quantities in the Castaing and Beck-Cohen methods ( $\varepsilon_{r}$ and $\beta_{d}$, respectively) are correlated as a power-law, through Bayes' theorem the two methods can be seen to be the same (see Eq. (18)). With the surrogate definition of $\varepsilon_{r}$ as in Eq. (4) and a proper subsystem size (Section IVB), we find that $\beta_{d}$ and $\varepsilon_{r}$ exhibit a power-law relation, as Fig. [6]llustrates. In this sense, the Castaing and Beck-Cohen methods describe the same PDF of $\delta v_{r}$ through the different conditional values which are correlated. Our experimental observation of a relation $\beta_{d} \propto\left(\varepsilon_{r}\right)^{-2 / 3}$ in Fig. 6] follows also 

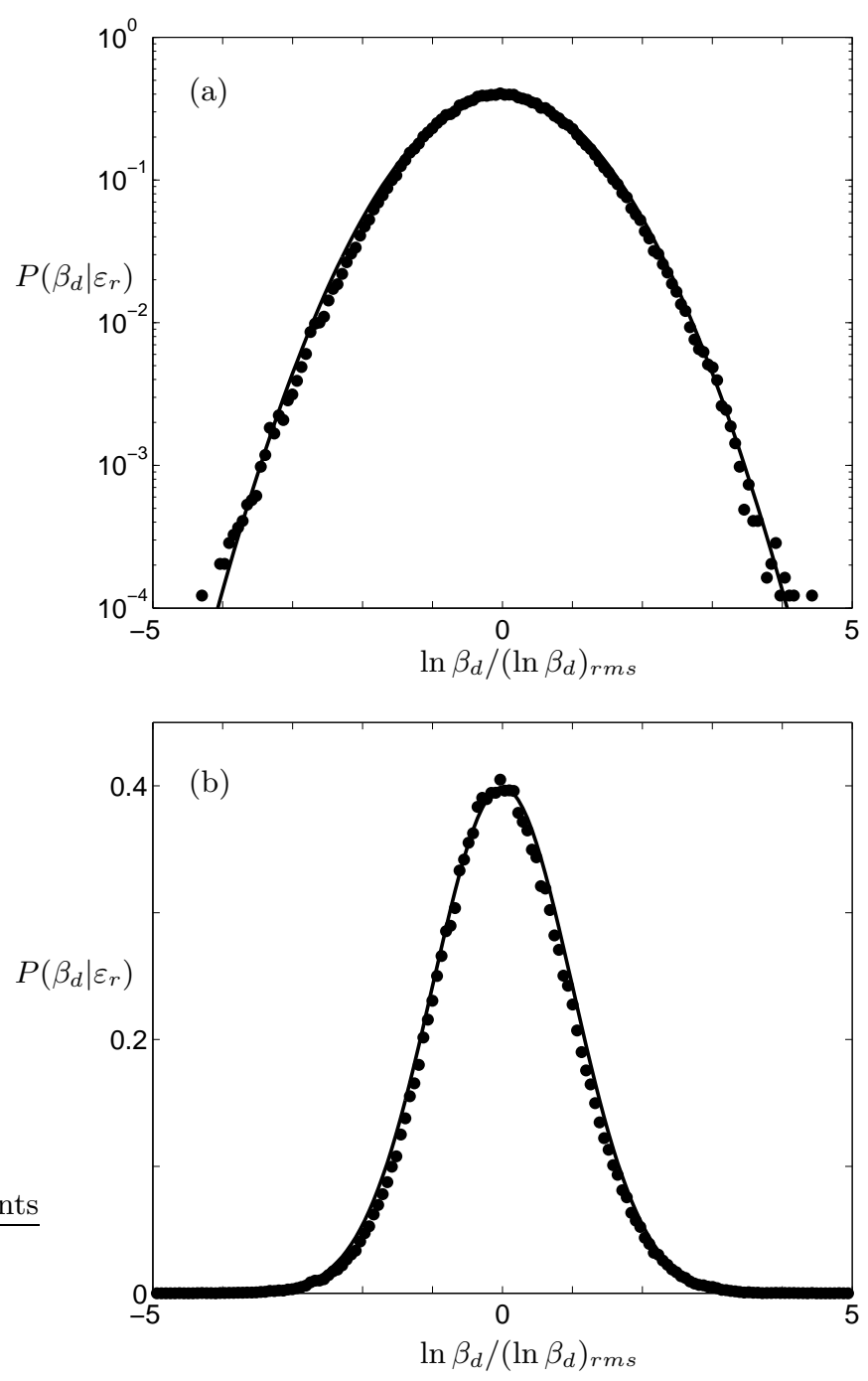

FIG. 7: The Gaussian distribution of $\ln \beta_{d}$ conditioned by $\varepsilon_{r}$, plotted on (a) $\log$ and (b) linear scales. The solid lines represent a Gaussian distribution of $\ln \beta_{d}$, that is, the $\log$ normal distribution of $\beta_{d}$. The dots represent the mean values of $\ln \beta_{d}$ from experiments.

from a dimensional analysis,

$$
\begin{aligned}
{\left[\beta_{d}\right]=} & {\left[\frac{T^{2}}{L^{2}}\right]=[L]^{-2 / 3} \times\left[\frac{L^{2}}{T^{3}}\right]^{-2 / 3} } \\
& \Rightarrow \beta_{d} \propto r^{-2 / 3} \varepsilon_{r}^{-2 / 3}
\end{aligned}
$$

where square brackets [.] denote the dimension of a physical quantity, $T$ is the dimension of time and $L$ is the dimension of length.

The probability of $\beta_{d}$ conditioned to $\varepsilon_{r}, P\left(\beta_{d} \mid \varepsilon_{r}\right)$, is log-normally distributed, as Fig. [7 illustrates. Our assumption in Eq. (9) holds with the surrogate $\varepsilon_{r}$ and $\beta_{d}$, where $d$ is properly chosen (Section IVB). Thus the integral of two log-normal distributions, $\int P\left(\beta_{d} \mid \varepsilon_{r}\right) P\left(\varepsilon_{r}\right) d \varepsilon_{r}$, is another log-normal distribution, $P\left(\beta_{d}\right)$. That is, if $P\left(\beta_{d} \mid \varepsilon_{r}\right)$ is a log-normal distribution with the mean of $\ln \varepsilon_{r}$, a $\log$-normal distribution of $\varepsilon_{r}$ in Castaing's method is equivalent with a log-normal distribution of $\beta_{d}$ in Beck-Cohen's method.

\section{CONCLUSIONS}

Both Castaing and Beck-Cohen methods have been very successful in describing the non-Gaussian distribution of velocity differences in turbulence [14, 50]. Although the relation of Beck-Cohen's method and Tsallis statistics [61] to turbulence has been questioned 50, 54, [55, 56], the fit to data is quite good [57, 58, 59, 60]. We have presented a method for determining subsystem size in the Beck-Cohen method, thus eliminating the need for a fitting parameter.

We have also shown that Castaing's method can be converted to Beck-Cohen method - the log-normal distribution of $\varepsilon_{r}$ in Castaing's method gives rise to a lognormal distribution of $\beta_{d}$ in Beck-Cohen's method. In that sense, the two methods describe the non-Gaussian distribution of $\delta v_{r}$ in the same way, $P\left(\delta v_{r}\right)=\int$ Gaussian distribution $\times \log$-normal distribution.

The authors thank C. Beck and E.G.D. Cohen for reading the manuscript and making helpful suggestions, and A.M. Reynolds and E. Sharon for helpful discussions. This research was supported by the Office of Naval Research. S. Jung acknowledges the support of a Donald D. Harrington fellows program.
[1] A. Kolmogorov, Dokl. Akad. Nauk SSSR 30, 299 (1941).

[2] U. Frisch, Turbulence, the Legacy of A.N. Kolmogorov (Cambridge University Press, 1995).

[3] C. W. Van Atta and W. Y. Chen, J. Fluids Mech. 44, 145 (1970).

[4] E. M. Lifshitz and L. D. Landau, Fluid Mechanics
(Butterworth-Heinemann, 1987), 2nd ed., the remark on the variation of energy dissipation appeared in the 1st ed. of book in 1944.

[5] G. Batchelor and A. Townsend, Proc. Roy. Soc. Lond. A 199, 238 (1949).

[6] H. Grant, R. Stewart, and A. Moilliet, J. Fluid Mech. 
12, 241 (1962).

[7] H. Grant, R. Stewart, and A. Moilliet, J. Fluid Mech. 224, 429 (1991).

[8] A. Kolmogorov, J. Fluid Mech. 13, 82 (1962).

[9] A. Obukhov, J. Fluid Mech. 13, 77 (1962).

[10] G. Pedrizzetti, E. Novikov, and A. Praskovsky, Phys. Rev. E 53, 475 (1996).

[11] H. Fujisaka, Y. Nakayama, T. Watanabe, and S. Grossmann, Phys. Rev. E 65, 046307 (2002).

[12] S. Chen, K. Sreenivasan, and M. Nelkin, Physical Review Letters 79, 1253 (1997).

[13] T. Nakano, D. Fukayama, A. Bershadskii, and T. Gotoh, Journal of the Physical Society of Japan 71, 2148 (2002).

[14] B. Castaing, Y. Gagne, and E. Hopfinger, Physica D 46, 177 (1990).

[15] C. Beck and E. Cohen, Physica A 322, 267 (2003).

[16] G. S. Lewis and H. L. Swinney, Phys. Rev. E 59, 5457 (1999).

[17] B. Castaing, Y. Gagne, and M. Marchand, Physica D 68, 387 (1993).

[18] A. Arneodo, S. Manneville, and J. Muzy, Euro. Phys. J. B 1, 129 (1998).

[19] B. Castaing, Physica D 73, 31 (1994).

[20] B. Chabaud, A. Naert, J. Peinke, F. Chilla, B. Castaing, and B. Hebral, Phys. Rev. Lett. 73, 3227 (1994).

[21] B. Castaing and B. Dubrulle, J. Phys. II 5, 895 (1995).

[22] A. Arneodo, J. Muzy, and S. Roux, J. Phys. II 7, 363 (1997).

[23] P. Chainais, P. Abry, and J. Pinton, Phys. Fluids 11, 3524 (1999).

[24] H. Kahalerras, Y. Malecot, Y. Gagne, and B. Castaing, Phys. Fluids 10, 910 (1998).

[25] A. Arneodo, N. Decoster, and S. Roux, Phys. Rev. Lett. 83, 1255 (1999).

[26] J. Pinton, F. Plaza, L. Danaila, P. Le Gal, and F. Anselmet, Physica D 122, 187 (1998).

[27] L. Sorriso-Valvo, V. Carbone, P. Veltri, G. Consolini, and R. Bruno, Geophysical Research Letters 26, 1801 (1999).

[28] P. Kailasnath, K. Sreenivasan, and G. Stolovitzky, Phys. Rev. Lett. 68, 2766 (1992).

[29] P. Tabeling, G. Zocchi, F. Belin, J. Maurer, and H. Willaime, Phys. Rev. E 53, 1613 (1996).

[30] P. Belin, F. Tabeling and H. Willaime, Physica D 93, 52 (1996).

[31] E. S. C. Ching and K. L. Chau, Phys. Rev. E 63, 047303 (2001).

[32] Y. Gagne, M. Marchand, and B. Castaing, J. Phys. II 4, 1 (1994).

[33] G. Stolovitzky, P. Kailasnath, and K. Sreenivasan, Phys. Rev. Lett. 69, 1178 (1992).
[34] L. Wang, S. Chen, J. Brasseur, and J. Wyngaard, J. Fluid Mech. 309, 113 (1996).

[35] R. Stewart, J. Wilson, and R. Burling, J. Fluid Mech. 41, 141 (1970).

[36] M. Fisher and P. O. A. L. Davies, J. Fluid Mech. 18, 97 (1964).

[37] O. Chanal, B. Chabaud, B. Castaing, and B. Hebral, Euro. Phys. J. B 17, 309 (2000).

[38] J. Elsner and W. Elsner, Measurement Science and Technology 7, 1334 (1996).

[39] J. C. Wyngaard and H. Tennekes, Phys. Fluids 13, 1962 (1970).

[40] J.-F. Pinton and R. Labbé, J. Phys. II 4, 1461 (1994).

[41] J. Wyngaard and S. Clifford, J. Atmos. Sci. 34, 922 (1977).

[42] R. Antonia, N. Phan-Yhien, and A. Chambers, J. Fluid Mech. 100, 193 (1980).

[43] J. Lumley, Phys. Fluids 8, 1056 (1965).

[44] F. Champagne, J. Fluid Mech. 86, 67 (1978).

[45] M. Nelkin, Advances in Physics 43, 143 (1994).

[46] C. Tsallis, J. Stat. Phys. 52, 479 (1988).

[47] S. Gille and S. Smith, Journal Of Physical Oceanography 30, 125 (2000).

[48] S. Smith and S. Gille, Phys. Rev. Lett. 81, 5249 (1998).

[49] C. Beck, Europhys. Lett. 64, 151 (2003).

[50] C. Beck, Physica D 193, 195 (2004).

[51] D. Lathrop, J. Fineberg, and H. L. Swinney, Phy. Rev. A 46, 6390 (1992).

[52] S. Rizzo and A. Rapisarda, in Experimental Chaos: 8th Experimental Chaos Conference, edited by S. Boccaletti (AIP, Florence, Italy, 2004), pp. 176-181.

[53] A. Turiel, G. Mato, N. Parga, and J. Nadal, Phys. Rev. Lett. 80, 1098 (1998).

[54] T. Gotoh and R. H. Kraichnan, Physica D 193, 231 (2004).

[55] M. Nauenberg, Phys. Rev. E 67, 036114 (2003).

[56] C. Tsallis, Physica D 193, 3 (2004).

[57] S. Jung, B. Storey, J. Aubert, and H. Swinney, Physica D 193, 252 (2004).

[58] K. E. Daniels, C. Beck, and E. Bodenschatz, Physica D 193, 208 (2004).

[59] N. Mordant, A. M. Crawford, and E. Bodenschatz, Physica D 193, 245 (2004).

[60] C. N. Baroud and H. L. H.L. Swinney, Physica D 183, 21 (2003).

[61] Beck and Cohen have shown that their method includes Tsallis statistics and other statistics [15]. A log-normal distribution is indistinguishable from Tsallis statistics except in long tails [50]. 